\title{
Gait characteristics of persons with bilateral transtibial amputations
}

\author{
Po-Fu Su, MS; ${ }^{1-2 *}$ Steven A. Gard, PhD; ${ }^{1-5}$ Robert D. Lipschutz, CP; ${ }^{5-6}$ Todd A. Kuiken, MD, PhD ${ }^{1,3,6}$ \\ ${ }^{1}$ Department of Biomedical Engineering, Robert R. McCormick School of Engineering and Applied Science, Northwest- \\ ern University, Evanston, IL; ${ }^{2}$ Northwestern University Prosthetics Research Laboratory and Rehabilitation Engineering \\ Research Program, Chicago, IL; ${ }^{3}$ Department of Physical Medicine and Rehabilitation, Feinberg School of Medicine, \\ Northwestern University, Chicago, IL; ${ }^{4}$ Jesse Brown Department of Veterans Affairs Medical Center, Chicago, IL; \\ ${ }^{5}$ Northwestern University Prosthetics-Orthotics Center, Chicago, IL; ${ }^{6}$ Rehabilitation Institute of Chicago, Chicago, IL
}

\begin{abstract}
The gait characteristics of persons with unilateral transtibial amputations are fairly well documented in the literature. However, much less is known about the gait of persons with bilateral transtibial amputations. This study used quantitative gait analysis to investigate the gait characteristics of 19 persons with bilateral transtibial amputations. To reduce variability between subjects, we fitted all subjects with Seattle Lightfoot II feet 2 weeks before their gait analyses. The data indicated that subjects walked with symmetrical temporospatial, kinematic, and kinetic parameters. Compared with nondisabled controls, the subjects with amputations walked with slower speeds and lower cadences, had shorter step lengths and wider step widths, and displayed hip hiking during swing phase. Additionally, compared with the nondisabled controls walking at comparable speeds, the subjects with amputations demonstrated reduced ankle dorsiflexion and knee flexion in stance phase, reduced peak ankle plantar flexor moment, reduced positive ankle power (i.e., energy return) in late stance, and increased positive and negative hip power. These results demonstrate the deficiencies in current prosthetic componentry and suggest that further research is needed to enhance prosthesis function and improve gait in persons with amputations.
\end{abstract}

Key words: amputee, bilateral, gait, hip hiking, kinematics, kinetics, prosthesis, rehabilitation, step width, transtibial, walking speed.

\section{INTRODUCTION}

An estimated 358,000 people with lower-limb amputations live in the United States [1]. Of these, approxi- mately 220,000 have transtibial amputations and 11,400 have bilateral transtibial amputations. During walking, persons with bilateral transtibial amputations have been reported to expend approximately 40 to 120 percent more energy per unit distance than nondisabled persons [2-4]. Currently, our understanding of the unique needs of persons with bilateral transtibial amputations is limited since no quantitative gait analyses of their gait characteristics have been published. If more is known about their gait mechanics, people with bilateral transtibial amputations will be better served by modern prosthetics.

Investigations that have performed quantitative gait analyses of persons with unilateral transtibial amputations typically report that these persons walk at slower speeds and lower cadences and with shorter step lengths compared with nondisabled persons [5-8]. Gait asymmetries are common in persons with unilateral amputations and are evident by a relatively longer step length, a shorter

Abbreviations: GRF $=$ ground reaction force, MAC $=$ Motion Analysis Corporation, SD = standard deviation, VA = Department of Veterans Affairs, VACMARL = VA Chicago Motion Analysis Research Laboratory.

* Address all correspondence to Po-Fu Su, MS; Northwestern University Prosthetics Research Laboratory and Rehabilitation Engineering Research Program, 345 E Superior St, RIC 1441, Chicago, IL 60611; 312-238-6500; fax: 312238-6510. Email: p-su@northwestern.edu

DOI: 10.1682/JRRD.2006.10.0135 
stance time, and a longer swing time on the prosthetic side [7,9-10]. The sagittal-plane ankle motion on the prosthetic side of persons with transtibial amputations is generally smaller than that of nondisabled persons [11]. Peak stance-phase knee flexion on the prosthetic side is typically reduced compared with nondisabled persons [6,12]. During prosthetic swing phase, persons with unilateral transtibial amputations sometimes exhibit hip hiking [13], a compensatory motion in which the pelvis is lifted on the prosthetic side to increase foot clearance. The magnitude of the first peak of the vertical ground reaction force (GRF) on the sound limb is generally larger than that of the prosthetic limb [11,14]. Peak internal ankle plantar flexion moments and peak positive and negative ankle joint powers of the prosthesis are reduced in persons with transtibial amputations [15-16]. Many of the gait characteristics observed in persons with unilateral transtibial amputations are also present in persons with bilateral transtibial amputations.

The inferior walking performance of persons with amputations is often attributed to the current state of prosthetic technology [11-12,14,16-17]. This conclusion is based on gait studies of persons with unilateral transtibial amputations. However, persons with unilateral transtibial amputations can compensate with their sound leg to some extent during walking, which can make discerning deficiencies in the prosthesis and identifying areas where significant improvement is needed extremely difficult. Because persons with bilateral transtibial amputations have a reduced ability to compensate, studying their gait may help researchers better identify shortcomings in prosthesis function and improve prosthetic component design.

In this study, we used quantitative gait analysis to characterize the walking pattern of persons with bilateral transtibial amputations. The knowledge gained will benefit prosthetists when prescribing prostheses to this population and help researchers better understand the limitations of current prosthetic technology.

\section{METHODS}

\section{Subjects and Prosthetic Components}

We recruited 19 subjects from clinics and prosthetics fitting centers in the Chicago metropolitan area. The criteria for selecting subjects were broad because of the small population of persons with bilateral transtibial amputations who walk with prostheses. The inclusion criteria included that the subject be a minimum of 2 years postamputation and an independent, functional ambulator with no serious medical conditions or physical symptoms of musculoskeletal, cardiac, or other significant health issues. We did not place restrictions on subject age, weight, height, or residual-limb length. The study prosthetist evaluated each subject's prosthetic sockets to ensure adequate fit before admission to the study. All subjects signed informed consent forms that were approved by the Northwestern University Institutional Review Board.

At the beginning of the study, an experienced, certified prosthetist fitted all the subjects with amputations with Seattle Lightfoot II feet (Seattle Systems, Poulsbo, Washington) with appropriate keel stiffness that was selected based on the subject's weight and activity level. The Seattle Lightfoot II uses a Delrin keel and is a commonly used low-profile dynamic response foot. We conducted a quantitative gait analysis 2 weeks later so that the subjects could sufficiently acclimate to the prosthetic feet.

\section{Gait Data Acquisition}

Data collection and analyses for the study were conducted in the Department of Veterans Affairs (VA) Chicago Motion Analysis Research Laboratory (VACMARL) (Jesse Brown VA Medical Center, Chicago, Illinois). The VACMARL has an eight-camera Eagle Digital Real-Time motion capture system (Motion Analysis Corporation [MAC], Santa Rosa, California) that measures and quantifies marker movements. We used a modified Helen Hayes marker set to define each participant's biomechanical model. As the subject walked along the walkway, the positions of the markers were recorded by the motion analysis cameras mounted around the periphery of the room. Six force platforms (Advanced Mechanical Technology, Inc; Watertown, Massachusetts) located midway along the walkway and embedded flush with the floor measured GRFs. The kinematic and kinetic data were collected with EVa Real-Time (MAC). The kinematic data were acquired at $120 \mathrm{~Hz}$, and the kinetic data were simultaneously recorded at a $960 \mathrm{~Hz}$ sampling rate. We used the GRF and motion data to calculate joint moments and powers via inverse dynamics using OrthoTrak software (MAC). 
During the gait analysis, the subjects were initially instructed to ambulate at their freely selected walking speed, then their fastest comfortable speed, and finally their slowest comfortable speed. Data from a total of 10 to 15 trials were collected for each walking speed, and the subjects were allowed to rest at any time during the experiment.

\section{Data Analysis}

Missing data points were interpolated with a cubicspline technique. The raw data were then filtered by a fourth-order bidirectional Butterworth infinite-impulse response digital filter with an effective cut-off frequency of $6.0 \mathrm{~Hz}$. OrthoTrak software calculated temporospatial data, joint angles, GRFs, joint moments, and powers. We developed customized MATLAB (The MathWorks, Natick, Massachusetts) programs to calculate mean and standard deviation (SD) values for the gait parameters and to generate figures.

Data acquired from the subjects with amputations were compared with data from 14 nondisabled persons on file in a laboratory database. Gait speed is a critical variable, and individuals with amputations generally walk slower than nondisabled individuals. Therefore, we compared the slow walking speed of the nondisabled controls with the freely selected walking speed of the subjects with amputations to mitigate differences due to speed alone. SPSS (SPSS Inc, Chicago, Illinois) was used for statistical analysis of the speed-matched data; an independent samples $t$-test with the level of statistical significance set at $p<0.05$ was used.

\section{RESULTS}

A total of 19 subjects with bilateral transtibial amputations were enrolled in the study. Their average age was 52.8 years ( $\mathrm{SD} \pm 17.6$ years). Their average height and mass were $171.9 \mathrm{~cm}$ and $77.4 \mathrm{~kg}$, respectively. The average age, height, and mass of the 14 nondisabled controls were 26 years, $174.2 \mathrm{~cm}$, and $72.3 \mathrm{~kg}$, respectively.

Four of the subjects with amputations used a singlepoint cane on their right side to assist walking during their gait analyses; all other subjects walked without an assistive device. While ambulating, these four subjects always held the cane in the right hand and in contact with the ground during the left stance phase. The results showed that all the subjects, including the four that used a cane, demonstrated reasonably good symmetry during gait (Table 1) and had similar vertical GRF magnitudes for both legs. The four subjects who used a cane did not seem to rely appreciably on it to support their body weight. They probably used the cane to provide a sense of security, improve stability, and prevent falling rather than to support a significant amount of body weight during walking. Based on this observation, only the subjects' right side data were analyzed.

\section{Temporospatial Data}

The mean \pm SD freely selected walking speed of the subjects with amputations was $0.9 \pm 0.3 \mathrm{~m} / \mathrm{s}$, which was significantly slower than that of the nondisabled controls,

Table 1.

Temporospatial data (mean \pm standard deviation) for subjects with bilateral transtibial amputations and nondisabled controls walking at various speeds. Gray columns indicate speed-matched comparisons. Double support time is average of two periods of double support times in gait cycle.

\begin{tabular}{|c|c|c|c|c|c|c|}
\hline \multirow[b]{2}{*}{ Variable } & \multicolumn{3}{|c|}{ Amputation } & \multicolumn{2}{|c|}{ Nondisabled } & \multirow[b]{2}{*}{$p$-Value } \\
\hline & Slow & $\begin{array}{c}\text { Freely } \\
\text { Selected }\end{array}$ & Fast & Slow & $\begin{array}{c}\text { Freely } \\
\text { Selected }\end{array}$ & \\
\hline Right Step Length (cm) & $45.1 \pm 9.8$ & $57.3 \pm 11.5$ & $64.8 \pm 13.5$ & $57.8 \pm 7.4$ & $70.5 \pm 7.4$ & 0.88 \\
\hline Left Step Length (cm) & $45.7 \pm 11.0$ & $56.5 \pm 12.4$ & $64.3 \pm 15.0$ & $58.6 \pm 7.1$ & $67.6 \pm 10.3$ & 0.55 \\
\hline Cadence (step/min) & $74.1 \pm 14.2$ & $93.8^{*} \pm 13.7$ & $106.1 \pm 15.1$ & $84.3^{*} \pm 10.7$ & $104.3 \pm 10.6$ & 0.04 \\
\hline Right Stance Time (\% gait cycle) & $67.4 \pm 8.5$ & $64.8 \pm 4.3$ & $63.2 \pm 4.0$ & $65.1 \pm 1.1$ & $62.2 \pm 2.4$ & 0.77 \\
\hline Left Stance Time (\% gait cycle) & $68.8 \pm 5.4$ & $64.2 \pm 3.7$ & $62.5 \pm 3.8$ & $65.2 \pm 1.5$ & $61.1 \pm 2.3$ & 0.30 \\
\hline Double Support Time (\% gait cycle) & $19.3 \pm 3.7$ & $14.5 \pm 3.7$ & $12.9 \pm 4.8$ & $15.1 \pm 2.0$ & $11.5 \pm 3.6$ & 0.56 \\
\hline
\end{tabular}


who walked at $1.2 \pm 0.2 \mathrm{~m} / \mathrm{s}(p<0.01)$. To compare the gait analysis results of the subjects with amputations and the nondisabled group, we speed-matched the data. Gait data were not specifically acquired from the nondisabled controls walking at the freely selected speed of the subjects with amputations. Instead, we noticed that the freely selected walking speed of the subjects with amputations was not significantly different from the nondisabled controls' slow speed $(0.8 \mathrm{~m} / \mathrm{s}, p=0.3)$. The speed-matched comparison indicated that the subjects with amputations displayed higher cadences ( $p=0.04$, Table 1) and wider step widths $(p<0.01)$, but the step lengths between the two groups were comparable $(p=0.75)$. The single and double support times were comparable between the subjects with amputations and the nondisabled controls.

\section{Gait Kinematics}

The ankle dorsiflexion angle of the subjects with amputations was reduced during middle and late stance phases compared with the nondisabled controls $(p<0.01$, Table 2, Figure 1). The amount of stance-phase knee flexion of the subjects with amputations was also significantly less than that of the nondisabled controls $(p=0.02)$, but the swing-phase knee flexion was comparable $(p=0.23$, Figure 2). The subjects with amputations displayed greater peak-to-peak hip motions than the nondisabled controls ( $p<0.01$, Figure 3 ). Greater pelvic obliquity during swing phase was observed in the subjects with amputations ( $p=0.11$, Figure 4 ) and the phase appeared to be shifted.

\section{Gait Kinetics}

The following kinetic parameters were analyzed: magnitude of the first peak of the vertical GRF, peak fore-aft acceleration GRF, peak fore-aft deceleration GRF, peak ankle plantar flexion moment, peak ankle dor- siflexion moment, peak ankle power absorption, peak ankle power "generation," peak hip power absorption, and peak hip power generation (Table 3).

The vertical GRF curves of the subjects with amputations were similar to those of the nondisabled controls (Figure 5). The posterior-directed portion of the fore-aft GRF is often described as the "braking" or deceleration force associated with deceleration of the body center of mass, and the anterior-directed portion is referred to as the "propulsive" or acceleration force associated with forward acceleration of the body center of mass. For the fore-aft GRFs, the subjects with amputations displayed a trend of lower peak deceleration and acceleration forces than the nondisabled controls (Figure 6), but the differences were not statistically significant. The peak ankle plantar flexion and dorsiflexion moments were significantly smaller in the subjects with amputations compared with the nondisabled controls ( $p<0.01$, Figure 7$)$. The peak negative powers were comparable in the ankle power curve, but the peak positive power of the subjects with amputations was only about one-fourth the magnitude of that observed in the nondisabled controls $(p<$ 0.01 , Figure 8). The subjects with amputations demonstrated significantly greater positive and negative hip joint power magnitudes than the nondisabled controls $(p<0.01$ and $p=0.03$, respectively, Figure 9).

\section{DISCUSSION}

Freely selected walking speed is a commonly measured gait parameter and is often used as an indicator of overall walking performance. The freely selected walking speed of the subjects with bilateral transtibial amputations in this study $(0.9 \mathrm{~m} / \mathrm{s})$ was much slower than that of the nondisabled controls $(1.2 \mathrm{~m} / \mathrm{s})$. Similar trends in persons

Table 2.

Peak-to-peak kinematic data (mean \pm standard deviation) for subjects with bilateral transtibial amputations and nondisabled controls walking at various speeds. Gray columns indicate speed-matched comparisons.

\begin{tabular}{|c|c|c|c|c|c|c|}
\hline \multirow{2}{*}{ Variable } & \multicolumn{3}{|c|}{ Amputation } & \multicolumn{2}{|c|}{ Nondisabled } & \multirow{2}{*}{$p$-Value } \\
\hline & Slow & Freely Selected & Fast & Slow & Freely Selected & \\
\hline$\overline{\text { Ankle Plantar/Dorsiflexion, Stance }\left(^{\circ}\right)}$ & $10.3 \pm 4.1$ & $12.5^{*} \pm 3.1$ & $13.9 \pm 3.7$ & $20.2^{*} \pm 3.5$ & $19.5 \pm 3.6$ & $<0.01$ \\
\hline Knee Flexion/Extension, Gait Cycle $\left(^{\circ}\right)$ & $58.7 \pm 12.3$ & $64.9 \pm 11.8$ & $69.0 \pm 11.7$ & $61.7 \pm 4.1$ & $67.7 \pm 5.4$ & 0.23 \\
\hline Hip Flexion/Extension $\left({ }^{\circ}\right)$ & $38.9 \pm 7.6$ & $43.6^{*} \pm 6.9$ & $47.8 \pm 8.4$ & $36.5^{*} \pm 2.1$ & $43.1 \pm 3.1$ & $<0.01$ \\
\hline Pelvic Obliquity, Coronal Plane $\left(^{\circ}\right)$ & $9.3 \pm 3.0$ & $8.4 \pm 2.8$ & $8.7 \pm 4.4$ & $6.3 \pm 2.1$ & $8.2 \pm 2.3$ & 0.11 \\
\hline
\end{tabular}




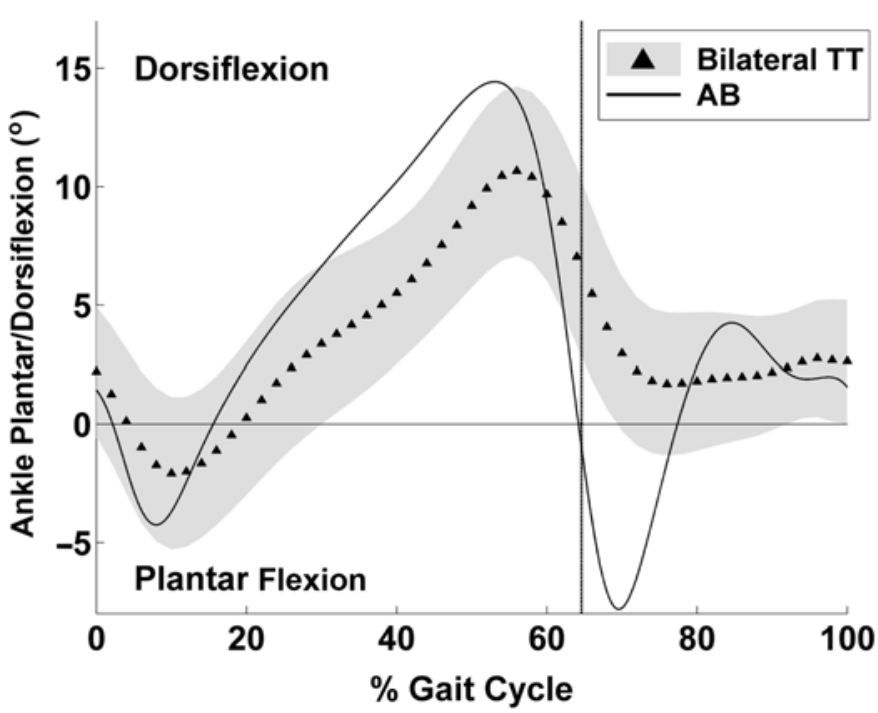

Figure 1.

Mean pattern of sagittal-plane ankle joint angles for subjects with bilateral transtibial amputations (Bilateral TT) walking at $0.9 \mathrm{~m} / \mathrm{s}$ and nondisabled controls (AB) walking at $0.8 \mathrm{~m} / \mathrm{s}$. Shaded area on either side of Bilateral TT mean represents 1 standard deviation. Vertical line represents toe-off.



Figure 2.

Mean pattern of sagittal-plane knee joint angle for subjects with bilateral transtibial amputations (Bilateral TT) walking at $0.9 \mathrm{~m} / \mathrm{s}$ and nondisabled controls (AB) walking at $0.8 \mathrm{~m} / \mathrm{s}$. Shaded area on either side of Bilateral TT mean represents 1 standard deviation. Vertical line represents toe-off.

with unilateral transtibial amputations have been reported in the literature. By comparison, persons with unilateral transtibial amputations reportedly walk at speeds of about 1.1 to $1.4 \mathrm{~m} / \mathrm{s}[6,18]$. Therefore, the persons with bilateral

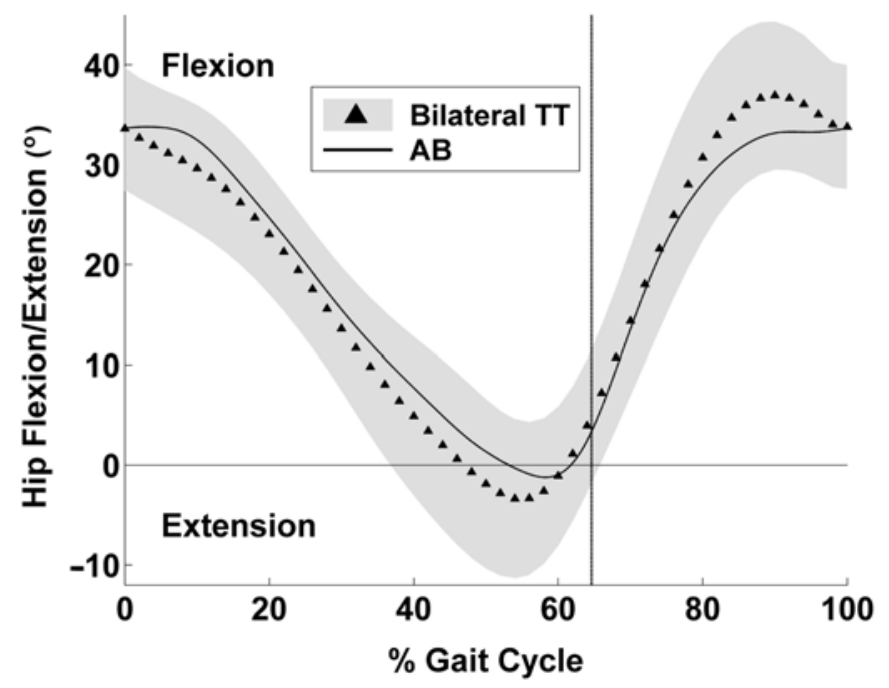

Figure 3.

Mean pattern of sagittal-plane hip joint angles for subjects with bilateral transtibial amputations (Bilateral TT) walking at $0.9 \mathrm{~m} / \mathrm{s}$ and nondisabled controls (AB) walking at $0.8 \mathrm{~m} / \mathrm{s}$. Shaded area on either side of Bilateral TT mean represents 1 standard deviation. Vertical line represents toe-off.

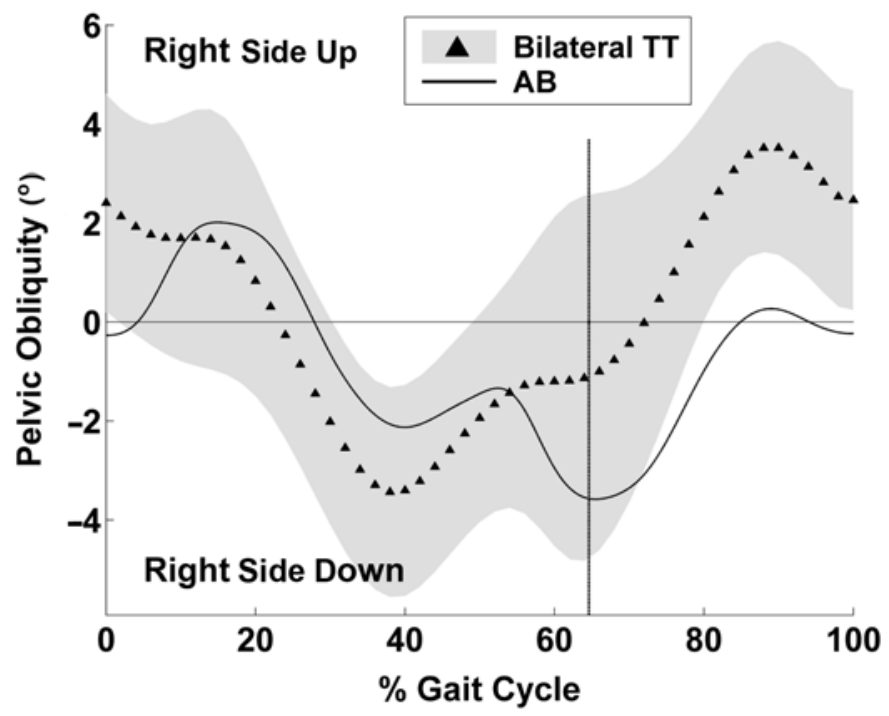

Figure 4.

Mean pattern of pelvic obliquity angles for subjects with bilateral transtibial amputations (Bilateral TT) walking at $0.9 \mathrm{~m} / \mathrm{s}$ and nondisabled controls (AB) walking at $0.8 \mathrm{~m} / \mathrm{s}$. Shaded area on either side of Bilateral TT mean represents 1 standard deviation. Vertical line represents toe-off.

amputations in this study walked slower than their unilateral counterparts. The freely selected walking speeds for these different groups indicate that persons with unilateral amputations can compensate for their limb loss to some 
JRRD, Volume 44, Number 4, 2007

Table 3.

Kinetic data (mean \pm standard deviation) for subjects with bilateral transtibial amputations and nondisabled controls walking at various speeds. Ground reaction forces (GRFs) were normalized by body weight (BW). Gray columns indicate speed-matched comparisons.

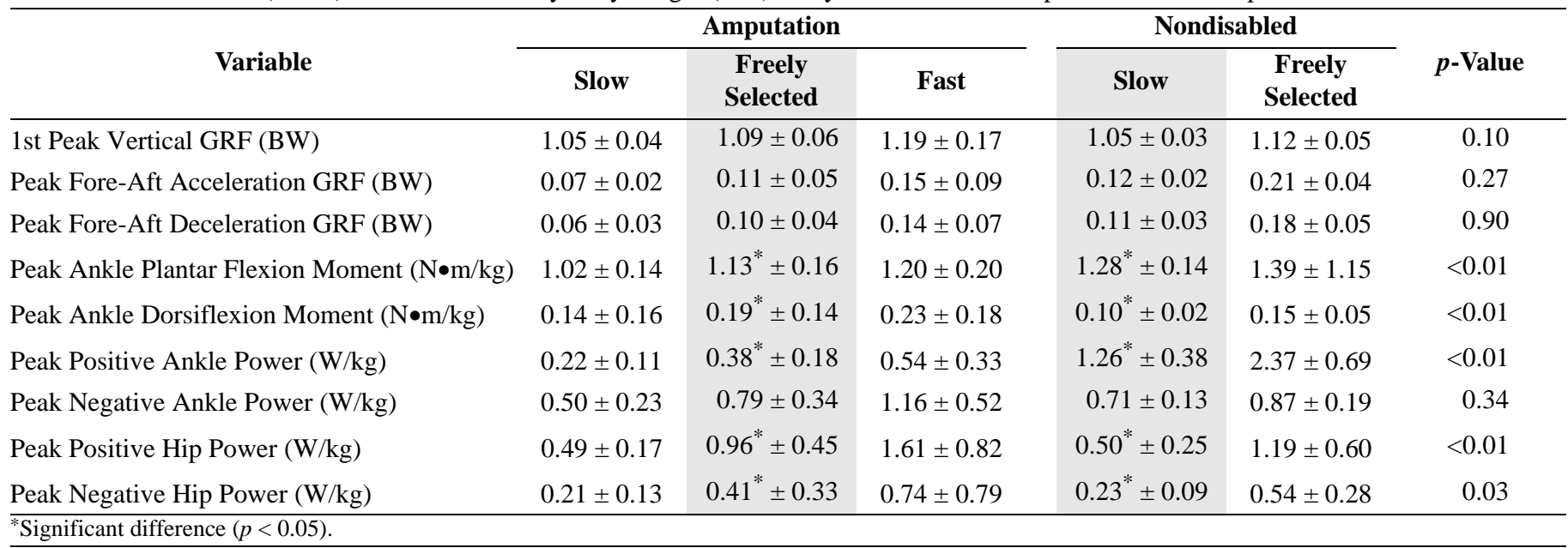

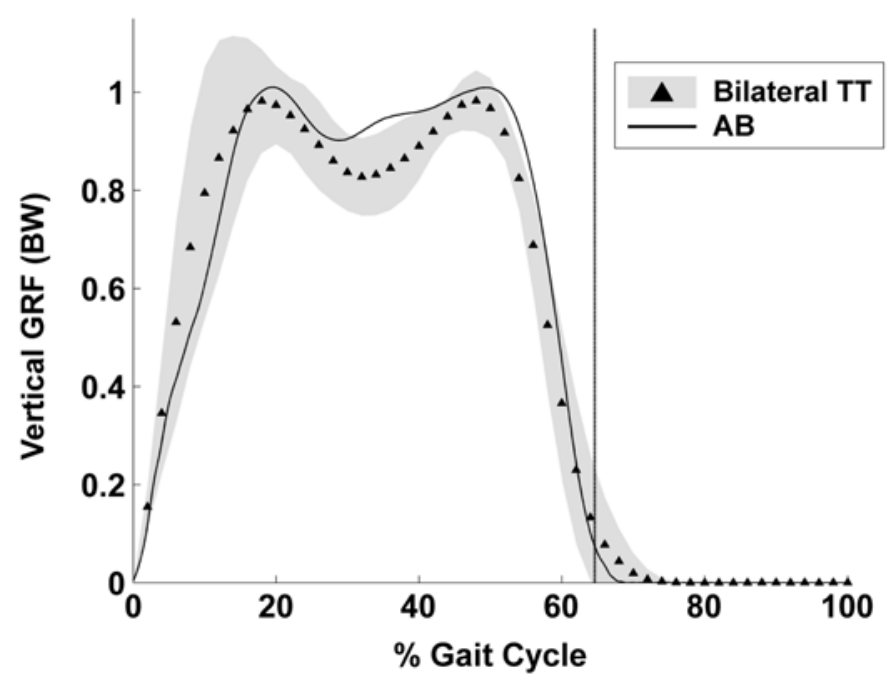

Figure 5.

Mean pattern of vertical ground reaction force (GRF) for subjects with bilateral transtibial amputations (Bilateral TT) walking at $0.9 \mathrm{~m} / \mathrm{s}$ and nondisabled controls $(\mathrm{AB})$ walking at $0.8 \mathrm{~m} / \mathrm{s}$. GRFs were normalized by body weight (BW). Shaded area on either side of Bilateral TT mean represents 1 standard deviation. Vertical line represents toe-off.

extent by relying more on their sound leg and, thus, are able to accommodate for some of the deficiencies associated with the prosthesis. Further investigation into the increased reliance that persons with unilateral amputations place on their sound leg seems warranted.

To reduce the effect of walking speed on differences in the temporospatial, kinematic, and kinetic data, we compared the data from the freely selected speed of the

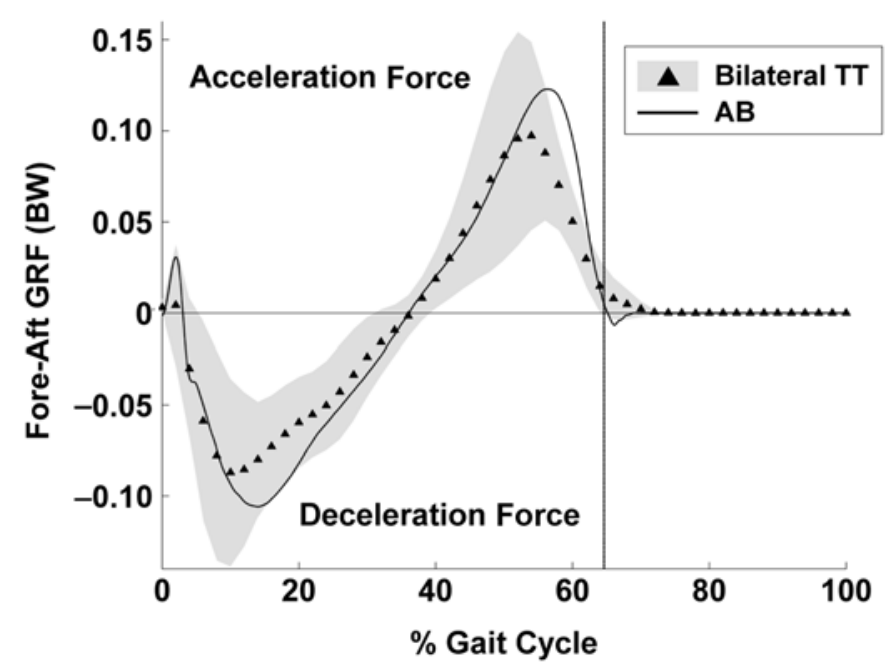

Figure 6.

Mean pattern of fore-aft ground reaction force (GRF) for subjects with bilateral transtibial amputations (Bilateral TT) walking at $0.9 \mathrm{~m} / \mathrm{s}$ and nondisabled controls $(\mathrm{AB})$ walking at $0.8 \mathrm{~m} / \mathrm{s}$. GRFs were normalized by body weight (BW). Shaded area on either side of Bilateral TT mean represents 1 standard deviation. Vertical line represents toe-off.

subjects with amputations with that of the nondisabled controls walking at their slow comfortable speed. Although the speeds were similar, the nondisabled group walked approximately $0.08 \mathrm{~m} / \mathrm{s}$ slower than the amputation group, which may have affected statistical significance for some parameters.

In the speed-matched data, higher cadences were observed in the subjects with amputations compared with 


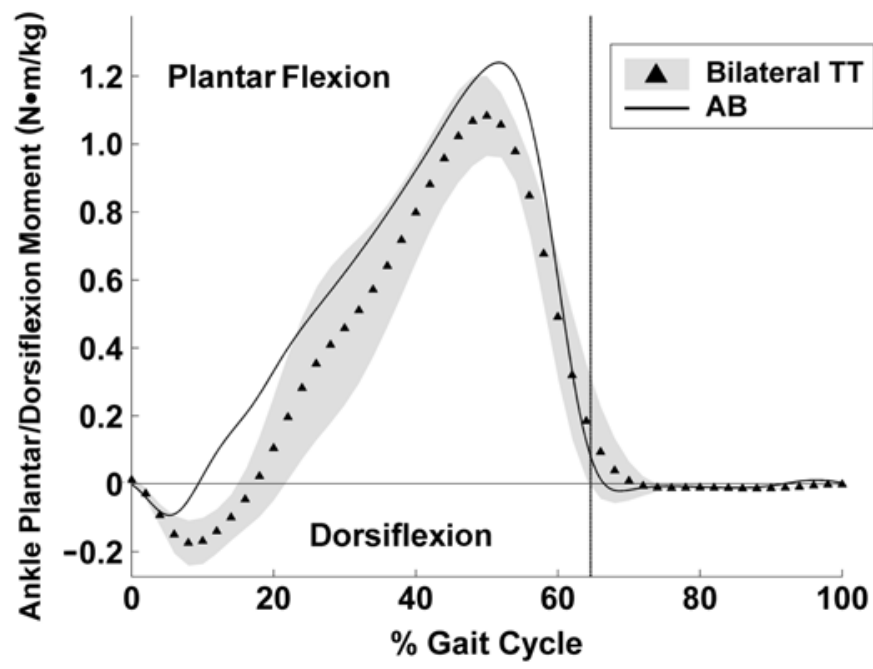

Figure 7.

Mean pattern of ankle flexion/extension moment for subjects with bilateral transtibial amputations (Bilateral TT) walking at $0.9 \mathrm{~m} / \mathrm{s}$ and nondisabled controls $(\mathrm{AB})$ walking at $0.8 \mathrm{~m} / \mathrm{s}$. Shaded area on either side of Bilateral TT mean represents 1 standard deviation. Vertical line represents toe-off.

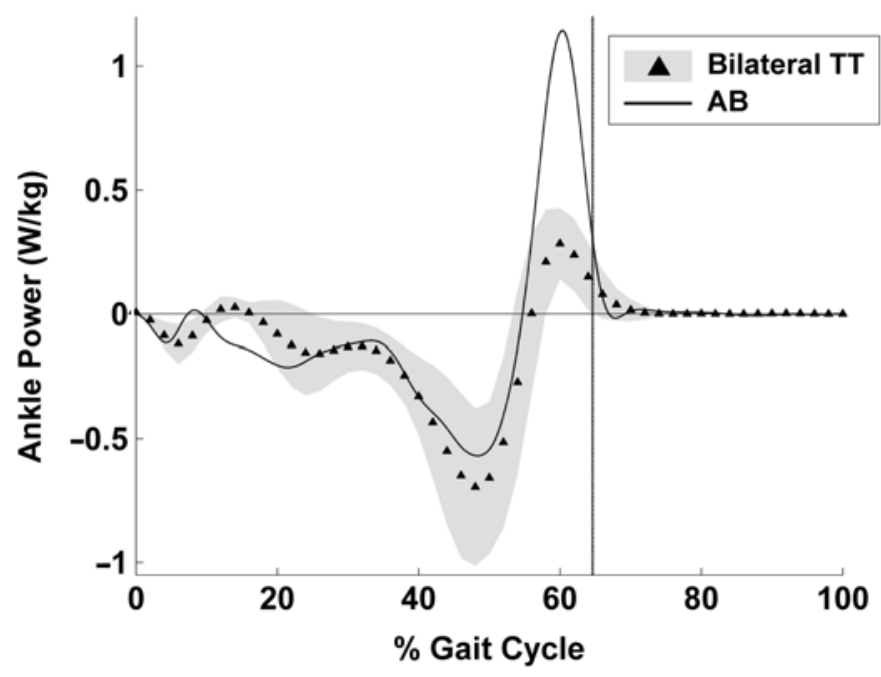

Figure 8.

Mean pattern of ankle power for subjects with bilateral transtibial amputations (Bilateral TT) walking at $0.9 \mathrm{~m} / \mathrm{s}$ and nondisabled controls (AB) walking at $0.8 \mathrm{~m} / \mathrm{s}$. Shaded area on either side of Bilateral TT mean represents 1 standard deviation. Vertical line represents toe-off.

the nondisabled controls. The two groups' step lengths were nearly equal, even though the freely selected walking speed of the subjects with amputations was slightly faster than the slow speed of the nondisabled controls. If

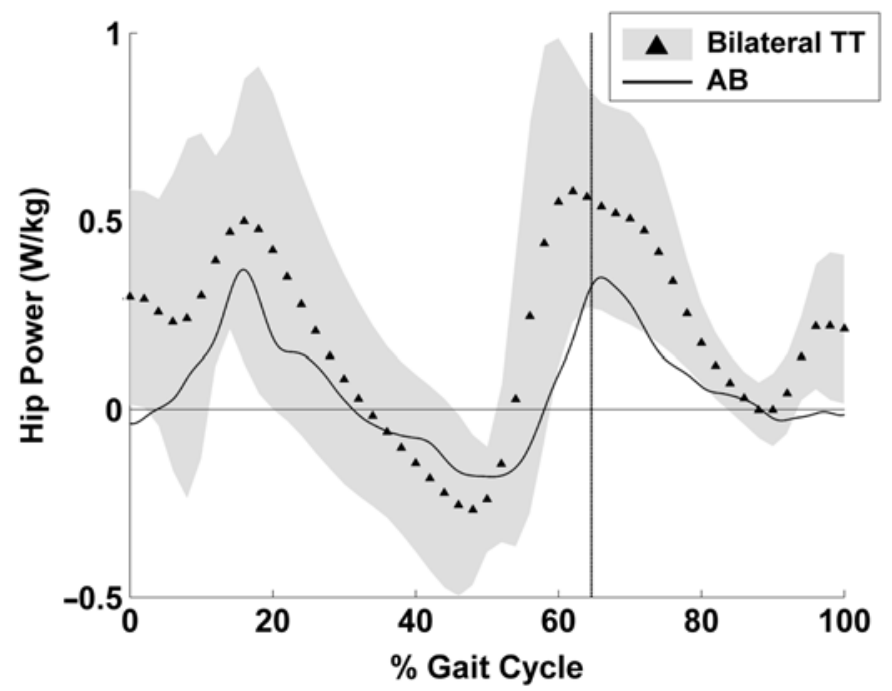

Figure 9.

Mean pattern of hip power for subjects with bilateral transtibial amputations (Bilateral TT) walking at $0.9 \mathrm{~m} / \mathrm{s}$ and nondisabled controls (AB) walking at $0.8 \mathrm{~m} / \mathrm{s}$. Shaded area on either side of Bilateral TT mean represents 1 standard deviation. Vertical line represents toe-off.

both groups had walked at precisely the same speed, the subjects with amputations might have displayed higher cadences and shorter step lengths compared with the nondisabled controls. Step length may have been relatively shortened in the subjects with amputations by their lack of dynamic plantar flexion during terminal stance phase. However, this trend was not observed in studies of individuals with unilateral transtibial amputations [6,11].

Persons with inferior dynamic balance generally walk with greater lateral trunk motion and adopt an increased step width to enhance stability [19]. Step width of the subjects with amputations was about 50 percent wider than that of the nondisabled controls during gait. The subjects with amputations may have adopted wider step widths and shorter step lengths to reduce the likelihood of a fall, which indicates that they have poorer stability and less perceived security than nondisabled persons. Somewhat surprisingly, our subjects with amputations demonstrated stance- and swing-phase durations comparable with those of the nondisabled controls. This finding contrasts with studies of persons with unilateral transtibial amputations [7,9-10], who typically demonstrate a longer stance phase, shorter swing phase, and shorter step length on the sound side compared with the prosthetic side. Clearly, persons with unilateral transtibial amputations have better stability during gait when 
their body weight is supported by the sound leg. However, subjects with bilateral transtibial amputations have better symmetry, which undoubtedly affects the different phase durations of gait.

The data indicate that the prosthetic feet provided less ankle motion during the stance phase of walking than was used by the nondisabled controls (Table 2, Figure 1). The Seattle Lightfoot II does not have an articulating ankle joint, so the measured ankle joint plantar flexion motion during early stance phase was primarily due to the compression of the heel, while the dorsiflexion motion during mid- to late stance phase resulted from the bending of the keel of the prosthetic foot. The prosthetic foot is intended to replace the lost anatomical foot and ankle functions, incorporating attributes such as joint simulation, shock absorption, a stable weight-bearing base of support, muscle simulation, and cosmesis [20]. The foot should maintain contact with the ground in late stance as the body progresses forward while load is transferred to the contralateral leg. Ankle function was not sufficiently restored when the subjects with amputations walked with the nonarticulated prosthetic feet used in this study. The lack of active control in the prostheses may also contribute to the difference in "ankle motions," particularly in late stance phase. Prosthetic foot and/or ankle mechanisms that provide appropriate ankle motion with proper stiffness may allow subjects with amputations to walk with increased stability and greater efficiency.

The Seattle Lightfoot II was chosen because it is a dynamic response foot with a solid ankle that may be used with additional components to permit multiaxial motion at the ankle. The data presented in this article were actually collected as baseline information for a more comprehensive study on the effect of prosthetic ankle mechanisms in persons with bilateral transtibial amputations. Quantitative gait analyses were performed on the subjects when they were initially fitted with the Seattle Lightfoot II without prosthetic ankle units and eventually when they walked with torsion units and components that provided ankle plantar flexion and dorsiflexion. (The results from the study of subjects with bilateral transtibial amputations walking with the prosthetic ankle units will be presented in a subsequent article.)

The dorsiflexion offset observed in the swing-phase data of the subjects with amputations was due to their prosthetic alignment (Figure 1), which is biased toward slight dorsiflexion. This alignment enables the subjects to be more stable statically since it encourages the center of pressure under the feet to shift forward and the GRF vector to shift anterior to the anatomical knee joint axis. Furthermore, this dorsiflexed prosthetic alignment is often necessary for improved standing stability in persons with bilateral transtibial amputations since they could not dorsiflex their ankles if the GRF vector were to inadvertently shift posteriorly and throw them off balance. Care must be taken, however, to ensure that this initial dorsiflexion angle does not create an excessive external knee flexion moment during initial contact and loading response phase of gait or permit excessive knee flexion (drop-off) at terminal stance.

Several degrees of prosthetic ankle plantar flexion motion were observed in the group mean data of the subjects with amputations immediately following toe-off (Figure 1). However, analysis of the prosthetic ankle-motion data from each individual indicated no prosthetic ankle motion after toe-off. Toe-off was found to occur at different times for the different subjects. Therefore, the apparent prosthetic ankle motion shown in Figure 1 was an anomaly caused by the averaging of data from all the subjects.

Stance-phase knee flexion plays a critical role in shock absorption during the loading response phase [21]. Perry claimed that controlled ankle plantar flexion during loading response facilitates stance-phase knee flexion by pulling the shank forward [22]. The persons with bilateral transtibial amputations in this study walked with less peak-to-peak stance-phase knee flexion than the nondisabled individuals walking at similar speeds. Other studies have reported decreased stance-phase knee flexion in persons with unilateral transtibial amputations [12,23]. The subjects with amputations may have attempted to reduce stance-phase knee flexion to minimize relative motion between the residual limb and the prosthetic socket and to avoid high, uncomfortable pressures by maintaining the GRF vector orientation nearly coincident with the longitudinal axis of their residual limb.

The subjects with amputations lifted their pelvis on the swing side during gait, a compensatory action known as hip hiking. Hip hiking during the prosthetic swing phase is often observed in persons with unilateral transtibial or transfemoral amputations and is believed to be a compensatory motion that increases prosthetic foot clearance because of the inability to dorsiflex the prosthetic ankle [13]. However, hip hiking requires added metabolic energy to lift the mass of the body against gravity, thus increasing energy expenditure and reducing gait efficiency. Persons with bilateral transtibial amputations display bilateral hip hiking, which requires them to expend much more energy during gait compared with persons with unilateral amputations. 
The normal pattern of pelvic obliquity during the loading response phase of nondisabled walking is important for shock absorption [22,24]. The subjects with bilateral transtibial amputations raised the pelvis on the side of the trailing leg during the loading response phase instead of letting it drop, as observed in the nondisabled controls (Figure 4). Consequently, the shock absorbed during gait may have been substantially reduced in the subjects with amputations as a result of reduced stance-phase knee flexion and an abnormal pattern of pelvic obliquity. Loss of shock absorption could increase the magnitude of the GRF during gait and increase shock transmission to the body. Chronically reduced shock absorption in persons with unilateral transtibial amputations may contribute to the reported increases in osteoarthritis of the sound knee compared with the prosthetic limb and limbs of nondisabled persons [25]. Persons with bilateral transtibial amputations may be at increased risk for osteoarthritis in both knees because of their significantly reduced anatomical shock-absorption mechanisms.

The magnitude of the first peak of the vertical GRF typically indicates the capability of the locomotor system to absorb shock during gait. Previous studies of persons with unilateral transtibial amputations reported that the first peak of the vertical GRF on the prosthetic limb was about 5 percent higher than that of nondisabled persons when they walked at similar speeds, while the sound-side vertical GRF was about 15 percent greater than that of nondisabled persons [11,14]. Contrary to expectations, the speed-matched data from our study indicated that the first peak of the vertical GRFs of the subjects with bilateral transtibial amputations was not significantly different from that of the nondisabled controls. The magnitudes of the fore-aft GRF were also similar between the groups.

During early stance phase, the subjects with amputations displayed greater internal dorsiflexion moments than the nondisabled subjects (Figure 7). We observed that during the loading response phase, the vertical GRF magnitudes of the subjects with amputations were greater than those of nondisabled controls (Figure 5), which may have contributed to the greater ankle dorsiflexion moments. In contrast, smaller vertical GRFs of subjects with amputations during mid- to late stance phase appeared to produce smaller ankle plantar flexion moments. Winter and Sienko reported that the peak ankle plantar flexion moment of persons with unilateral transtibial amputations was only 60 to 70 percent of that of nondisabled individuals and suggested that this reduction was due to the absence of the ankle plantar flexors in persons with amputations [15].
Also possible is that the keel of the prosthetic foot was functionally shorter than that of the biological foot, reducing the moment arm between the GRFs and the ankle joint center during stance. Consequently, the ankle plantar flexion moments would be decreased.

The inverse dynamics calculations of joint moments and powers for the subjects with amputations used anthropometric data from nondisabled individuals. Custom data sets for the mass, center of mass position, and moment of inertia were not created for each amputated subject's prosthetic and residual-limb combinations. The complexity of making these types of measurements to create custom anthropomorphic data sets would have been time and cost prohibitive and probably would not have significantly improved the accuracy of the inverse dynamics calculations. In this study, the joint moment and power analyses focused on the stance phase of walking, when linear and angular accelerations of the limb segments are relatively small. Thus, errors in the inverse dynamic calculations are presumably small during stance phase. Caution is urged, however, against analysis of joint moments and powers of the swing phase of persons who walk with prostheses without consideration of custom anthropomorphic measures.

One should note that the prosthetic foot is passive and cannot generate any power. Therefore, the ankle joint "power" of the prosthesis presumably indicates the amount of energy stored and returned by the elastic deformation of the prosthetic foot. In terminal stance phase and early preswing, the forefoot of the Seattle Lightfoot II was loaded by body weight and deflected, as indicated by the ankle plantar flexion/dorsiflexion curve (Figure 1) and the ankle power curve (Figure 8). The energy stored in the keel was believed to be returned later in preswing, contributing to the positive peak ankle power. However, the subjects with bilaterial transtibial amputations exhibited a much lower peak power than the nondisabled controls in preswing, as has also been shown in persons with unilateral transtibial amputations [15-16]. Nondisabled individuals actively plantar flex at the end of stance phase, which is believed to provide push-off and generate significant power for forward progression. The absence of plantar flexors in subjects with bilateral transtibial amputations may have contributed to their observed smaller ankle power "generation” (i.e., energy return).

The subjects with amputations displayed greater hip power generation compared with the nondisabled individuals during early to midstance and at the time of toeoff (Figure 9). Increased hip power generation has been 
previously reported in several studies of persons with unilateral transtibial amputations [9,15,26]. Persons with amputations may use greater hip power generation to compensate for the work ordinarily performed by ankle plantar flexors prior to toe-off in nondisabled persons. The excessive work performed by the hip may further contribute to the increased energy expenditure often reported for amputee walking.

Comparing data at similar walking speeds is a common practice in quantitative gait analysis and identifies and highlights disparate movement patterns between different groups. One concern about the use of data from the slow walking speed of nondisabled individuals is that this gait pattern may not necessarily reflect their most energyefficient gait. However, data from Waters and Yakura indicate that nondisabled individuals can walk across a relatively broad range of speeds with little variation in their metabolic energy cost (i.e., energy expended per unit distance) [27]. Only at speeds outside their range of comfortable speeds does the energy cost begin to increase significantly. Comparing freely selected gait data between the group with amputations and the control group would alleviate the concern about data representing the most energy-efficient gait pattern, but these analyses would undoubtedly indicate significant differences in the gait parameters because of the two groups' dissimilar walking speeds. Therefore, though our method of comparing data between the two groups may be subject to some debate, we remain convinced that this approach is superior to other alternatives.

The average age of the subjects with amputations was significantly older than that of the nondisabled controls. However, investigators have previously pointed out that the pattern of adult gait is generally maintained from the second through the seventh decades of life in the absence of any underlying neurological conditions [28]. Therefore, we attribute the differences observed between the subjects with amputations and the control group to the bilateral amputations and not to age. Nonetheless, creating a control group with a similar mean age (and weight, height, etc.) as the experimental group would generally be recommended since doing so would eliminate concerns about extraneous independent variables weakening a study's internal validity.

The temporospatial, kinematic, and kinetic parameters of persons with bilateral transtibial amputations were found to be similar to those of the prosthetic side of per- sons with unilateral transtibial amputations. The observed range of prosthetic "ankle" motion was smaller than that of nondisabled subjects, but whether this contributes to the inferior walking performance in persons with transtibial amputations is not currently known. Having a prosthetic ankle joint or a prosthetic foot with a lower stiffness that permits greater motion might enhance the walking performance of the subjects with amputations, but this remains to be determined. Incorporating components that provide increased ankle motion in prostheses may allow persons with amputations to have improved function during gait that better approximates that of nondisabled persons and ultimately reduces the energy required to walk.

\section{CONCLUSIONS}

The subjects with bilateral transtibial amputations in this study walked with slower speeds, lower cadences, and shorter step lengths compared with the nondisabled individuals. When the two groups walked at similar speeds, the subjects with amputations displayed less stance-phase ankle dorsiflexion, less stance-phase knee flexion, reduced peak ankle plantar flexion moment, and less ankle power generation. During gait, the subjects with amputations also displayed bilateral hip hiking, a compensatory action that may have increased energy expenditure. Additional studies must further identify prosthetic deficiencies and ultimately develop solutions for improving gait in persons with amputations.

\section{ACKNOWLEDGMENTS}

Data for this project were acquired in the VACMARL of the Jesse Brown VA Medical Center, Chicago, Illinois.

This material was based on work supported by the National Institutes of Health, National Institute of Child Health and Human Development, grant 1R01HD42592. These contents are solely the responsibility of the authors and do not necessarily represent the official views of the National Institute of Child Health and Human Development.

The authors have declared that no competing interests exist. 


\section{REFERENCES}

1. National Health Interview Survey. Hyattsville (MD): National Center for Health Statistics, Centers for Disease Control and Prevention, U.S. Department of Health and Human Services; 2001.

2. DuBow LL, Witt PL, Kadaba MP, Reyes R, Cochran V. Oxygen consumption of elderly persons with bilateral below knee amputations: Ambulation vs wheelchair propulsion. Arch Phys Med Rehabil. 1983;64(6):255-59. [PMID: 6860095]

3. Gonzalez EG, Corcoran PJ, Reyes RL. Energy expenditure in below-knee amputees: Correlation with stump length. Arch Phys Med Rehabil. 1974;55(3):111-19. [PMID: 4817680]

4. Waters RL, Perry J, Antonelli D, Hislop H. Energy cost of walking of amputees: The influence of level of amputation. J Bone Joint Surg Am. 1976;58(1):42-46. [PMID: 1249111]

5. Prince F, Winter DA, Sjonnensen G, Powell C, Wheeldon RK. Mechanical efficiency during gait of adults with transtibial amputation: A pilot study comparing the SACH, Seattle, and Golden-Ankle prosthetic feet. J Rehabil Res Dev. 1998;35(2):177-85. [PMID: 9651889]

6. Torburn L, Perry J, Ayyappa E, Shanfield SL. Below-knee amputee gait with dynamic elastic response prosthetic feet: A pilot study. J Rehabil Res Dev. 1990;27(4):369-84. [PMID: 2089148]

7. Barth DG, Schumacher L, Sienko-Thomas S. Gait analysis and energy cost of below-knee amputees wearing six different prosthetic feet. J Prosthet Orthot. 1992;4(2):63-75.

8. Schmalz T, Blumentritt S, Jarasch R. Energy expenditure and biomechanical characteristics of lower limb amputee gait: The influence of prosthetic alignment and different prosthetic components. Gait Posture. 2002;16(3):255-63. [PMID: 12443950]

9. Sadeghi H, Allard P, Duhaime PM. Muscle power compensatory mechanisms in below-knee amputee gait. Am J Phys Med Rehabil. 2001;80(1):25-32. [PMID: 11138951]

10. Isakov E, Keren O, Benjuya N. Trans-tibial amputee gait: Time-distance parameters and EMG activity. Prosthet Orthot Int. 2000;24(3):216-20. [PMID: 11195356]

11. Snyder RD, Powers CM, Fontaine C, Perry J. The effect of five prosthetic feet on the gait and loading of the sound limb in dysvascular below-knee amputees. J Rehabil Res Dev. 1995;32(4):309-15. [PMID: 8770795]

12. Perry J, Boyd LA, Rao SS, Mulroy SJ. Prosthetic weight acceptance mechanics in transtibial amputees wearing the Single Axis, Seattle Lite, and Flex Foot. IEEE Trans Rehabil Eng. 1997;5(4):283-89. [PMID: 9422453]

13. Michaud SB, Gard SA, Childress DS. A preliminary investigation of pelvic obliquity patterns during gait in persons with transtibial and transfemoral amputation. J Rehabil Res Dev. 2000;37(1):1-10. [PMID: 10847567]
14. Powers CM, Torburn L, Perry J, Ayyappa E. Influence of prosthetic foot design on sound limb loading in adults with unilateral below-knee amputations. Arch Phys Med Rehabil. 1994;75(7):825-29. [PMID: 8024435]

15. Winter DA, Sienko SE. Biomechanics of below-knee amputee gait. J Biomech. 1988;21(5):361-67. [PMID: 3417688$]$

16. Gitter A, Czerniecki JM, DeGroot DM. Biomechanical analysis of the influence of prosthetic feet on below-knee amputee walking. Am J Phys Med Rehabil. 1991;70(3): 142-48. [PMID: 2039616]

17. Aleshinsky SY. An energy ‘sources' and 'fractions' approach to the mechanical energy expenditure problem-IV. Criticism of the concept of 'energy transfers within and between links.’ J Biomech. 1986;19(4):307-9. [PMID: 3711130]

18. Gard SA, Konz RJ. The effect of a shock-absorbing pylon on the gait of persons with unilateral transtibial amputation. J Rehabil Res Dev. 2003;40(2):109-24. [PMID: 15077637]

19. Murray MP, Sepic SB, Gardner GM, Mollinger LA. Gait patterns of above-knee amputees using constant-friction knee components. Bull Prosthet Res. 1980;10-34:35-45. [PMID: 7260459]

20. Kapp S, Cummings D. Transtibial amputation: Prosthetic management. In: Bowler JH, Michael JW, editors. Atlas of limb prosthetics. St. Louis (MO): Mosby-Year Book, Inc; 1992. p. 453-78.

21. Gard SA, Childress DS. The influence of stance-phase knee flexion on the vertical displacement of the trunk during normal walking. Arch Phys Med Rehabil. 1999;80(1): 26-32. [PMID: 9915368]

22. Perry J. Gait analysis: Normal and pathological function. Thorofare (NJ): SLACK; 1992.

23. Breakley JW. Gait of unilateral below-knee amputees. Orthot Prosthet. 1976;30(3):17-24.

24. Gard SA, Childress DS. The effect of pelvic list on the vertical displacement of the trunk during normal walking. Gait Posture. 1997;5(3):233-38.

25. Lemaire ED, Fisher FR. Osteoarthritis and elderly amputee gait. Arch Phys Med Rehabil. 1994;75(10):1094-99. [PMID: 7944914]

26. Schneider K, Hart T, Zernicke RF, Setoguchi Y, Oppenheim W. Dynamics of below-knee child amputee gait: SACH foot versus Flex foot. J Biomech. 1993;26(10): 1191-1204. [PMID: 8253824]

27. Waters RL, Yakura JS. The energy expenditure of normal and pathologic gait. Crit Rev Phys Rehabil Med. 1989;1(3): 183-209.

28. Blanke DJ, Hageman PA. Comparison of gait of young men and elderly men. Phys Ther. 1989;69(2):144-48. [PMID: 2913584]

Submitted for publication October 23, 2006. Accepted in revised form April 23, 2007. 